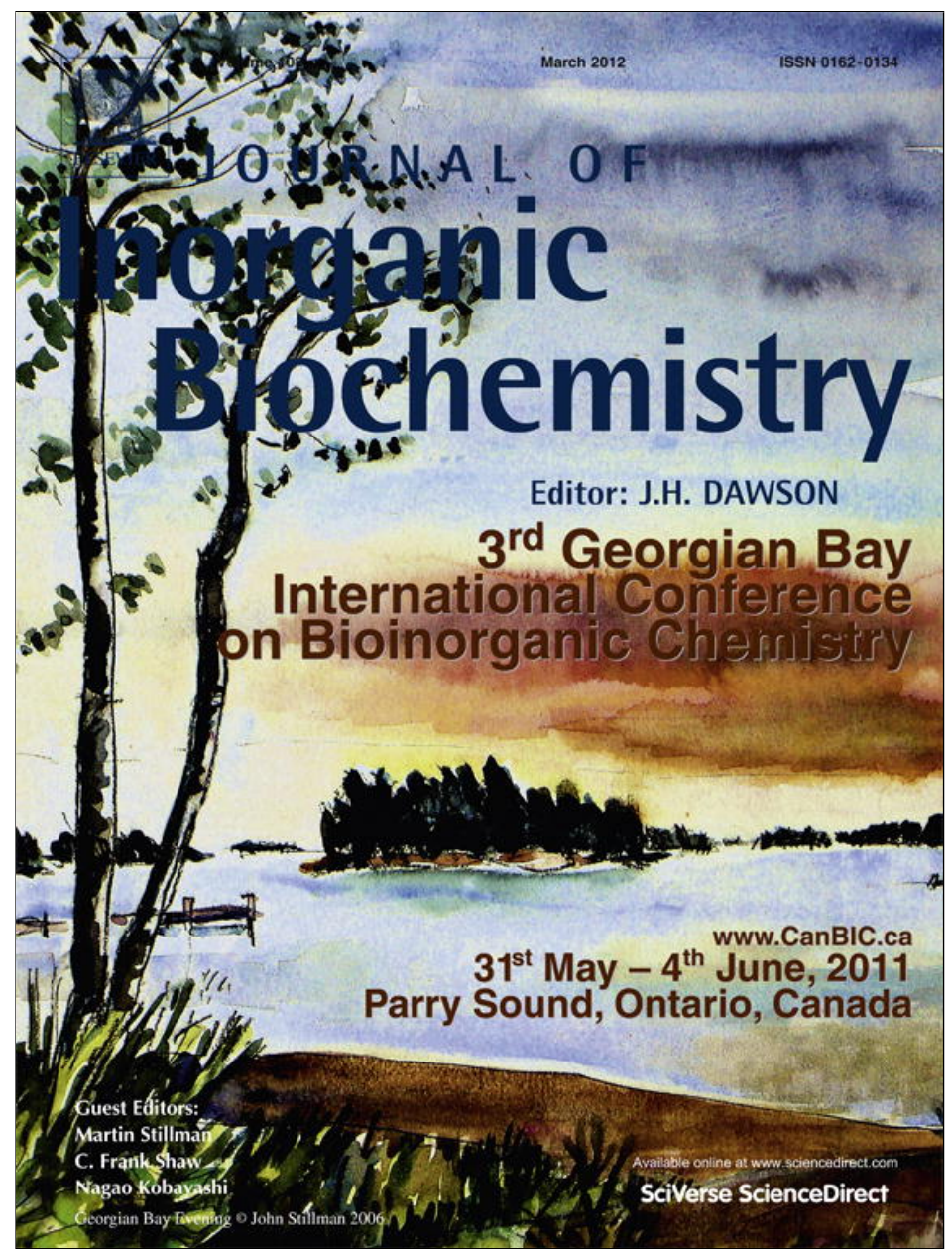

This article appeared in a journal published by Elsevier. The attached copy is furnished to the author for internal non-commercial research and education use, including for instruction at the authors institution and sharing with colleagues.

Other uses, including reproduction and distribution, or selling or licensing copies, or posting to personal, institutional or third party websites are prohibited.

In most cases authors are permitted to post their version of the article (e.g. in Word or Tex form) to their personal website or institutional repository. Authors requiring further information regarding Elsevier's archiving and manuscript policies are encouraged to visit: 


\title{
Novel Pt(II) and Pd(II) complexes with polyamine analogues: Synthesis and vibrational analysis
}

\author{
T.M. Silva ${ }^{\text {a,b,c }}$, S. Oredsson ${ }^{\text {b }}$, L. Persson ${ }^{\text {c }}$, P. Woster ${ }^{\text {d }}$, M.P.M. Marques ${ }^{\text {a,e,* }}$ \\ a Research Unit "Molecular Physical Chemistry", University of Coimbra, Portugal \\ ${ }^{\mathrm{b}}$ Department of Biology, University of Lund, Sweden \\ c Department of Experimental Medical Science, University of Lund, Sweden \\ d Department of Pharmaceutical and Biomedical Sciences, Medical University of South Carolina, SC, USA \\ e Department of Life Sciences, Faculty of Science and Technology, University of Coimbra, Portugal
}

\section{A R T I C L E I N F O}

\section{Article history:}

Received 26 August 2011

Received in revised form 10 November 2011

Accepted 23 November 2011

Available online 3 December 2011

\section{Keywords:}

Modified polyamines

$\mathrm{Pt}(\mathrm{II}) / \mathrm{Pd}(\mathrm{II})$ complexes

Raman spectroscopy

Infrared spectroscopy

Anticancer

\begin{abstract}
A B S T R A C T
A vibrational spectroscopy study (infrared and Raman) is reported for the biogenic polyamine analogues norspermidine (NSpd), $N^{1}, N^{11}$-bis(ethyl)norspermine (BENSpm) and $N^{1}$-cyclo-propylmethyl- $N^{11}$-ethylnorspermine (CPENSpm), as well as for their newly synthesised Pt(II) and Pd(II) complexes. Attending to the potential antineoplastic properties of this kind of systems, their full conformational characterization is essential for understanding the molecular basis of their cytotoxic activity and the mechanisms through which they are transported into the cell. The all-trans geometry was found to be favoured for all the alkylated polyamines, in their totally protonated state, while their polynuclear complexes presented a stable geometry very similar to that previously obtained for the analogous chelates with spermidine $\left(\mathrm{M}_{3} \mathrm{Spd}_{2}\right)$ and spermine $\left(\mathrm{M}_{2} \mathrm{Spm}\right)$, comprising two or three cisplatin-like $\left(\mathrm{MCl}_{2} \mathrm{NH}_{2}\right)$ moieties.
\end{abstract}

(c) 2011 Elsevier Inc. All rights reserved.

\section{Introduction}

Biogenic polyamines (PA's) - putrescine ( $\mathrm{Put}, \mathrm{H}_{2} \mathrm{~N}\left(\mathrm{CH}_{2}\right)_{4} \mathrm{NH}_{2}$ ), spermidine ( $\mathrm{Spd}, \mathrm{H}_{2} \mathrm{~N}\left(\mathrm{CH}_{2}\right)_{3} \mathrm{NH}\left(\mathrm{CH}_{2}\right)_{4} \mathrm{NH}_{2}$ ) and spermine (Spm, $\left.\mathrm{H}_{2} \mathrm{~N}\left(\mathrm{CH}_{2}\right)_{3} \mathrm{NH}\left(\mathrm{CH}_{2}\right)_{4} \mathrm{NH}\left(\mathrm{CH}_{2}\right)_{3} \mathrm{NH}_{2}\right)$ - are ubiquitous in cells of higher organisms, and essential for their growth and differentiation through tightly regulated processes [1,2]. Because of absolute polyamine requirement for cell proliferation, coupled to the positive correlation between polyamine levels and tumour growth, polyamines are regarded as a crucial target for anticancer treatment [3]. Numerous studies have focused on PA depletion by using synthetic antagonists, or by modulating specific enzymes associated to polyamine metabolism or degradation. Moreover, the chemotherapeutic effect of conventional chemotherapeutic agents may be assisted by this PA depletion in the tumour and host tissues, through the combined use of polyamine analogues which can even induce a synergistic effect with standard DNA-reactive cytotoxic drugs [4].

\footnotetext{
* Corresponding author at: Department of Life Sciences, Faculty of Science and Technology, University of Coimbra, Ap. 3046, 3001-401 Coimbra, Portugal. Tel.: + 351239826541 , fax : + 351239854448 .

E-mail address: pmc@ci.uc.pt (M.P.M. Marques).
}

Modified biogenic polyamines, in particular, have been shown to affect the regulatory paths associated to PA's biosynthesis, catabolism and transport, through a Trojan horse mechanism: the cell recognises these polyamine analogues as natural polyamines and they are promptly taken up and accumulated to high levels, thus stimulating PA catabolism while inhibiting the cellular uptake of biogenic PA's. Thus, the cell becomes depleted of these polyamines while being filled with the analogues which cannot take over their function [5]. Numerous PA analogues have lately been synthesised in view of their use in cancer chemotherapy [6] - either symmetrically or unsymmetrically alkylated (e.g. at the terminal nitrogens), conformationally restricted, or varying in the N-to-N alkyl bridges - and some of them have shown exceptional efficacy in animal tumour models coupled to surprisingly low toxicity in humans. The ethyl-substituted spermine analogue BENSpm $\left(N^{1}, N^{11}\right.$-bis(ethyl) norspermine), for instance, has shown a significant activity (both in vitro and in vivo) against human breast cancer cells [7,8], and is currently under Phase II of clinical trials [9]. This compound has also been found to exhibit a synergistic inhibitory effect on breast cancer cell proliferation in combination with 5-fluorouracil and paclitaxel [10], possibly through a novel antioestrogenic mechanism (via the oestrogen receptor $\alpha$ ).

Additionally, this type of linear alkylpolyamines is suitable ligands for metal ions such as Pt(II) and Pd(II), yielding complexes (namely polynuclear chelates) that were found to act as promising antineoplastic agents through a non-conventional (long-range interstrand) interaction with DNA, often displaying a higher efficacy than standard 
drugs (e.g. cisplatin, cis- $\left.\mathrm{Pt}\left(\mathrm{NH}_{3}\right)_{2} \mathrm{Cl}_{2}\right)$ [11,12]. However, although the transport, cellular uptake and activity of both the PA analogues and their metal chelates are known to be strongly dependent on their structural characteristics, these highly sensitive structure-activity relationships (SAR's) are very poorly known, which urges for a detailed analysis of this kind of systems at the molecular level.

Furthermore, in view of the promising results already obtained by the authors for bi- and trinuclear Pt(II) and Pd(II) chelates of spermidine and spermine regarding their interaction with DNA and antineoplastic properties [13-21], the present study on similar complexes with analogous PA's is of the utmost relevance with a view to optimize the anticancer activity of this kind of PA-based agents, in the light of their structural profile.

Therefore, this work has a twofold objective: i) the study of several modified polyamines by vibrational spectroscopic methods - Fourier transform infrared (FTIR) and Raman - coupled to quantum mechanical calculations, which, to the best of the authors' knowledge, have never been reported for these analogues-NSpd (norspermidine, $\left.\mathrm{H}_{2} \mathrm{~N}\left(\mathrm{CH}_{2}\right)_{3} \mathrm{NH}\left(\mathrm{CH}_{2}\right)_{3} \mathrm{NH}_{2}\right)$, BENSpm $\left(N^{1}, N^{11}\right.$-bis(ethyl)norspermine, $\left.\left(\mathrm{CH}_{3} \mathrm{CH}_{2}\right) \mathrm{HN}\left(\mathrm{CH}_{2}\right)_{3} \mathrm{NH}\left(\mathrm{CH}_{2}\right)_{3} \mathrm{NH}\left(\mathrm{CH}_{2}\right)_{3} \mathrm{NH}\left(\mathrm{CH}_{3} \mathrm{CH}_{2}\right)\right)$ and CPENSpm ( $N^{1}$-cyclopropyl-methyl- $N^{11}$-ethylnorspermine, $\quad\left(\mathrm{CH}_{2} \mathrm{C}_{3} \mathrm{H}_{5}\right) \mathrm{HN}\left(\mathrm{CH}_{2}\right)_{3}$ $\mathrm{NH}\left(\mathrm{CH}_{2}\right)_{3} \mathrm{NH}\left(\mathrm{CH}_{2}\right)_{3} \mathrm{NH}\left(\mathrm{CH}_{3} \mathrm{CH}_{2}\right)$ (Fig. 1); ii) the synthesis of new $\mathrm{Pt}(\mathrm{II})$ and Pd(II) complexes with NSpd and BENSpm, and their structural analysis by vibrational spectroscopy coupled to quantum mechanical calculations. The results will be compared and interpreted in the light of extensive spectroscopic and theoretical data previously obtained by the authors on the corresponding biogenic polyamines and their Pt(II) and $\operatorname{Pd}(\mathrm{II})$ chelates [22-31]. The influence of factors such as the type and number of metal centres, the nature of the ligands and the coordination pattern will be assessed. The knowledge thus gathered will be correlated with the corresponding biological profile - cytotoxicity and interference with polyamine homeostasis - assessed in a parallel study towards human breast cancer cells [T.M. Silva, S. Oredsson, L. Persson and M.P.M. Marques, manuscript in preparation], thus allowing to determine the molecular basis of this biological activity.

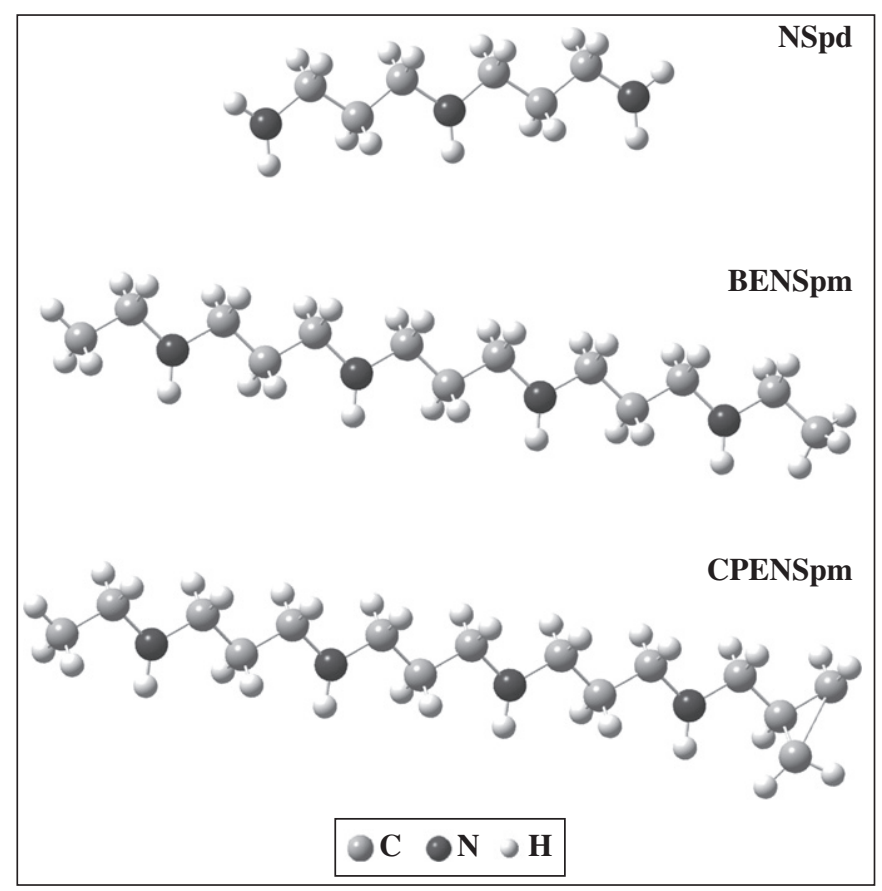

Fig. 1. Most stable structures calculated (B3LYP/6-31G**) for NSpd, BENSpm and CPENSpm.

\section{Experimental procedure}

\subsection{Chemicals}

$\mathrm{K}_{2} \mathrm{PtCl}_{4}$ (>99.9\%), $\mathrm{K}_{2} \mathrm{PdCl}_{4}$ (98\%) and NSpd trihydrochloride (98\%) were purchased from Sigma-Aldrich Chemical Co. (Sintra, Portugal). All chemicals and solvents were reagent grade and were used without further purification.

The PA analogues BENSpm tetrahydrochloride and CPENSpm tetrahydrobromide were made available by Dr Patrick Woster.

\subsection{Synthesis}

Novel Pt(II) and Pd(II) complexes with PA analogues were synthesised, according to optimised procedures based on previously reported methods for similar compounds (Pt(II) and Pd(II) chelates with biogenic polyamines $[21,32,33])$. The analogues used were either symmetrically or unsymmetrically substituted at the terminal nitrogens (BENSpm and CPENSpm, respectively), or alkylpolyamines varying in the N-to-N methylene bridges relative to the biogenic compound (NSpd) (Fig. 1). The chelates studied along this work were $\left(\mathrm{MCl}_{2}\right)_{3}(\mathrm{NSpd})_{2}(\mathrm{M}=\mathrm{Pt}(\mathrm{II})$ and $\mathrm{Pd}(\mathrm{II})),\left(\mathrm{PdCl}_{2}\right)_{2}(\mathrm{BENSpm})$ and $\left(\mathrm{PtCl}_{2}\right)_{2}$ (CPENSpm).

$\left(\mathrm{PdCl}_{2}\right)_{3}(\mathrm{NSpd})_{2}$ and $\left(\mathrm{PdCl}_{2}\right)_{2}(\mathrm{BENSpm})$ : Briefly, $2 \mathrm{mmol}$ of $\mathrm{K}_{2} \mathrm{PdCl}_{4}$ was dissolved in a minimal amount of water, and an aqueous solution containing 3 or $1 \mathrm{mmol}$ of the ligand (NSpd or BENSpm, respectively) was added dropwise under continuous stirring, the pH having been adjusted to $c a .8$ immediately after addition of the polyamine. After about 5-6 h stirring the solution was left to stand overnight. A yellow powder of the complex was obtained, which was filtered and washed repeatedly with cold acetone and ethanol. Yield: $\left(\mathrm{PdCl}_{2}\right)_{3}(\mathrm{NSpd})_{2}-81.2 \%$; $\left(\mathrm{PdCl}_{2}\right)_{2}(\mathrm{BENSpm})-88.6 \%$

$\left(\mathrm{PtCl}_{2}\right)_{3}(\mathrm{NSpd})_{2}$ and $\left(\mathrm{PtCl}_{2}\right)_{2}(\mathrm{CPENSpm})$. For the synthesis of the $\mathrm{Pt}(\mathrm{II})$ complexes a similar protocol was followed, starting from a solution containing 3 or $1 \mathrm{mmol}$ of the ligand (NSpd or CPENSpm, respectively) added (dropwise, under stirring) to a concentrated aqueous solution with $2 \mathrm{mmol}$ of $\mathrm{K}_{2} \mathrm{PtCl}_{4}$. Upon overnight rest a yellowish powder of the complex $\left(\left(\mathrm{PtCl}_{2}\right)_{3}(\mathrm{NSpd})_{2}\right.$ or $\left.\left(\mathrm{PtCl}_{2}\right)_{2}(\mathrm{CPENSpm})\right)$ was obtained, filtered and washed with cold acetone and ethanol. In this case, the $\mathrm{pH}$ was adjusted to $\mathrm{ca} .8$ and 12, respectively for the NSpd and CPENSpm chelates, after adding the ligand. Yield: $\left(\mathrm{PtCl}_{2}\right)_{3}(-$ Nspd $)_{2}-45.7 \%$; $\left(\mathrm{PtCl}_{2}\right)_{2}$ (CPENSpm) $-66.3 \%$.

The elemental analysis were carried out at the Central Analysis Laboratory and Chemistry Department (Mass Spectrometry Laboratory) of the University of Aveiro (Portugal) (C, N, H), and at the Elemental Analysis Laboratory of the University of Santiago de Compostela (Spain) (Pt, Pd). $\left(\mathrm{PtCl}_{2}\right)_{3}(\mathrm{Nspd})_{2}$-calculated-C: $13.6 \%$, H: 3.24\%, N: 7.92\%, Cl: 20.06\%, Pt: $55.19 \%$; found-C: $13.33 \%, \mathrm{H}: 3.16 \%, \mathrm{~N}: 7.89 \%$, Pt: $55.01 \%$. $\left(\mathrm{PdCl}_{2}\right)_{3}(\mathrm{Nspd})_{2}-$ calculated-C: $18.14 \%, \mathrm{H}: 4.32 \%, \mathrm{~N}: 10.58 \%, \mathrm{Cl}$ : 26.78\%, Pd: $40.18 \%$; found-C: $18.10 \%, \mathrm{H}: 4.20 \%, \mathrm{~N}: 10.32 \%$, Pt: 39.90\%. $\left(\mathrm{PdCl}_{2}\right)_{2}(\mathrm{BENSpm})-$ calculated-C: $26.06 \%, \mathrm{H}: 5.39 \%, \mathrm{~N}$ : 9.36\%, Cl: 23.67\%, Pd: $35.52 \%$; found-C: $25.88 \%, \mathrm{H}: 5.10 \%, \mathrm{~N}$ : 9.13\%, Pd: $35.23 \%$. $\left(\mathrm{PtCl}_{2}\right)_{2}$ (CPENSpm)-calculated-C: $22.45 \%, \mathrm{H}$ : 4.28\%, N: 6.98\%, Cl: $17.67 \%$, Pt: 48.62; found-C: $22.03 \%, \mathrm{H}$ : 4.12\%, N: $6.77 \%$, Pt: $48.10 \%$.

The complexes were further characterised by vibrational spectroscopy (FTIR and Raman-see discussion below).

\subsection{FTIR spectroscopy}

The Fourier transform infrared (FTIR) spectra were recorded in a Bruker Optics Vertex 70 FTIR spectrometer, in the range $400-4000 \mathrm{~cm}^{-1}$, using $\mathrm{KBr}$ discs ( $c a .2 \%(w / w))$, a $\mathrm{KBr}$ beamsplitter, and a liquid nitrogen cooled Mercury Cadmium Telluride (MCT) detector. The FTIR spectra were collected for $\mathrm{ca}$. 75 scans, with a $2 \mathrm{~cm}^{-1}$ resolution. The errors in wavenumbers were estimated to be less than $1 \mathrm{~cm}^{-1}$. 


\subsection{Raman spectroscopy}

The Raman spectra were obtained for the pure compounds, at room temperature, in a triple monochromator Jobin-Yvon T64000 Raman system (focal distance $0.640 \mathrm{~m}$, aperture $f / 7.5$ ) equipped with holographic gratings of 1800 grooves $\mathrm{mm}^{-1}$. The premonochromator stage was used in the subtractive mode. The detection system was a liquid nitrogen cooled non-intensified $1024 \times 256$ pixel ( $\left.1^{\prime \prime}\right)$ charge coupled device (CCD) chip.

The $514.5 \mathrm{~nm}$ line of an $\mathrm{Ar}^{+}$laser (Coherent, model Innova 300-05) was used as the excitation radiation, providing ca. $90 \mathrm{~mW}$ at the sample position. A $90^{\circ}$ geometry between the incident radiation and the collecting system was employed. The entrance slit was set to $200 \mu \mathrm{m}$, as well as the slit between the premonochromator and the spectrograph. Samples were sealed in Kimax glass capillary tubes of $0.8 \mathrm{~mm}$ inner diameter. Under the above mentioned conditions, the error in wavenumbers was estimated to be within $1 \mathrm{~cm}^{-1}$.

Room temperature Fourier transform Raman (FT-Raman) spectra were obtained in an RFS 100/S Bruker spectrophotometer. The $1064 \mathrm{~nm}$ line provided by a Nd:YAG laser was used, yielding $200 \mathrm{~mW}$ at the sample position. Resolution was set at $2 \mathrm{~cm}^{-1}$ and a $180^{\circ}$ geometry was employed.

\subsection{Quantum mechanical calculations}

The quantum mechanical calculations were performed using the GAUSSIAN 03 W programme [34] within the Density Functional Theory (DFT) approach, in order to properly account for the electron correlation effects (particularly important in this kind of systems). The widely employed hybrid method denoted by B3LYP, which includes a mixture of HF (Hartree-Foch) and DFT exchange terms and the gradientcorrected correlation functional of Lee, Yang and Parr [35,36], as proposed and parameterised by Becke [37,38], was used, along with the double-zeta split valence basis set 6-31G** [39].

Molecular geometries were fully optimised by the Berny algorithm, using redundant internal coordinates [40]: the bond lengths to within ca. $0.1 \mathrm{pm}$ and the bond angles to within $\mathrm{ca} .0 .1^{\circ}$. The final root-mean-square (rms) gradients were always less than $3 \times 10^{-4}$ hartree.bohr ${ }^{-1}$ or hartree.radian ${ }^{-1}$. No geometrical constraints were imposed on the molecules under study.

The harmonic vibrational wavenumbers, as well as Raman activities and infrared intensities, were also obtained, at the same theory level as the geometry optimization procedure. The widely employed scale factors of Merrick et al. [41] were used to reproduce the experimental data, leading to a quite good agreement between theoretically predicted and experimental frequencies.

\section{Results and discussion}

Linear alkylpolyamines, such as biogenic PA's spermidine and spermine or the presently investigated analogues, can adopt several geometries depending on the dihedral angles that determine their overall orientation, the most common ones being $60^{\circ}$ (gauche), $180^{\circ}$ (trans) and $-60^{\circ}$ (gauche $)$. At physiological $\mathrm{pH}$, these polyamines are polycations, all their nitrogen atoms being protonated, which hinders the formation of intramolecular $(\mathrm{N}) \mathrm{H}{ }^{\cdots} \mathrm{N}$ or $(\mathrm{C}) \mathrm{H}{ }^{\cdots}$ : $\mathrm{N}$ hydrogen bonds, the all-trans conformation being energetically favoured. In these conditions, only three effects are relevant in determining conformation: intermolecular $\mathrm{H}$-bonds, electrostatic and steric forces. Previous conformational analysis of $\alpha, \omega$-diamines $\left(\mathrm{H}_{2} \mathrm{~N}\left(\mathrm{CH}_{2}\right)_{n} \mathrm{NH}_{2}\right.$, $n=2$ to 10 and $n=12$ ), as well as of the larger spermidine (triamine) and spermine (tetramine) [42,22-27] allowed to corroborate that the all-trans is the predominant or even the sole conformation for this kind of totally extended polycationic molecules in the solid state. In fact, it was verified that, in the solid, intramolecular interactions are overruled by intermolecular ones. The most stable structures for the
PA analogues under study - NSpd, BENSpm and CPENSpm - are represented in Fig. 1. These were obtained through quantum mechanical methods, as the lowest energy geometries among the several possible conformations calculated for each molecule.

The corresponding vibrational analysis, carried out by both FTIR and Raman, allows to corroborate these conformational preferences. Interpretation of the experimental data was assisted by the calculated geometries and corresponding vibrational pattern, a quite good agreement having been obtained in all cases (Fig. 1S, Supplementary Material). Comparison of these results with the data previously reported [23-27] for the analogous biogenic polyamines spermidine and spermine (Figs. 1S and 2S, Supplementary Material) allows a clear assignment of the features corresponding to their common molecular core (Tables 1-3): $\mathrm{NH}_{2} / \mathrm{NH}_{3}$ deformation modes at about $1600 \mathrm{~cm}^{-1}$; intense features at $c a .1450-1500 \mathrm{~cm}^{-1}$ and $1200-1320 \mathrm{~cm}^{-1}$, mainly assigned to $\mathrm{CH}_{2}$ deformations (twisting and wagging respectively); strong signals predominantly due to $\mathrm{NH}_{2} / \mathrm{NH}_{3}$ deformations (also containing a contribution from $\mathrm{CH}_{2}$ deformations), detected at ca. 1260 and $1200 \mathrm{~cm}^{-1}$ for the solid

Table 1

Main vibrational modes $\left(\mathrm{cm}^{-1}\right)$ for $\mathrm{NSpd}, \mathrm{Pt}_{3}-\mathrm{NSpd}_{2}$ and $\mathrm{Pd}_{3}-\mathrm{NSpd}_{2}$.

\begin{tabular}{|c|c|c|c|c|c|c|c|}
\hline \multicolumn{3}{|l|}{ NSpd } & \multirow{2}{*}{\multicolumn{2}{|c|}{$\frac{\mathrm{Pt}_{3}-\mathrm{NSpd}_{2}}{\text { Exp. }}$}} & \multirow{2}{*}{\multicolumn{2}{|c|}{$\frac{\mathrm{Pd}_{3}-\mathrm{NSpd}_{2}}{\text { Exp. }}$}} & \multirow[t]{3}{*}{ Approximate description } \\
\hline \multicolumn{2}{|l|}{ Exp. } & \multirow[t]{2}{*}{ Calc. $^{a}$} & & & & & \\
\hline FTIR & Raman & & FTIR & Raman & FTIR & Raman & \\
\hline 3362 & 3362 & 3442 & 3262 & 3267 & 3269 & 3283 & $v_{s}\left(\mathrm{NH}_{3}\right)$ \\
\hline 3278 & 3302 & 3346 & 3248 & 3250 & 3224 & 3227 & $v_{\mathrm{s}}\left(\mathrm{NH}_{3}\right)+v_{\mathrm{s}}\left(\mathrm{NH}_{2}\right)$ \\
\hline \multirow[t]{2}{*}{3187} & 3188 & 3220 & 3215 & 3219 & 3172 & 3172 & $v_{\mathrm{as}}\left(\mathrm{NH}_{3}\right)+v_{\mathrm{as}}\left(\mathrm{NH}_{2}\right)$ \\
\hline & & 3217 & 3148 & 3152 & 3154 & 3158 & $v_{\mathrm{s}}\left(\mathrm{NH}_{2}\right)$ \\
\hline 2939 & 2955 & 3137 & 2942 & 2942 & 2939 & 2940 & $v_{\text {as }}\left(\mathrm{CH}_{2}\right)$ \\
\hline 2905 & 2909 & & 2877 & 2915 & 2874 & 2903 & $v_{\text {as }}\left(\mathrm{CH}_{2}\right)$ \\
\hline 2852 & 2861 & & 2854 & 2878 & 2846 & 2876 & $v_{\mathrm{s}}\left(\mathrm{CH}_{2}\right)$ \\
\hline 1600 & 1600 & & 1608 & & 1605 & & $\delta_{\text {sciss }}\left(\mathrm{NH}_{2}\right)$ \\
\hline 1466 & 1478 & 1470 & 1462 & 1459 & 1460 & 1459 & $\delta_{\text {sciss }}\left(\mathrm{CH}_{2}\right)$ \\
\hline 1439 & 1444 & 1410 & 1446 & & 1449 & & $\delta_{\text {sciss }}\left(\mathrm{CH}_{2}\right)$ \\
\hline 1365 & 1360 & & & & & & $\delta_{\text {sciss }}\left(\mathrm{CH}_{2}\right)$ \\
\hline \multirow[t]{2}{*}{1307} & 1300 & 1252 & 1258 & & 1249 & & $\delta_{\text {sciss }}\left(\mathrm{CH}_{2}\right)+\mathrm{t}\left(\mathrm{CH}_{2}\right)$ \\
\hline & & & 1172 & 1170 & 1171 & & $\begin{array}{l}\omega\left(\mathrm{CH}_{2}\right)+\mathrm{t}\left(\mathrm{CH}_{2}\right) \\
+\delta\left(\mathrm{NH}_{3}\right)+v(\mathrm{CC})\end{array}$ \\
\hline 1126 & 1113 & 1186 & 1148 & & 1146 & 1143 & $\begin{array}{l}\omega\left(\mathrm{CH}_{2}\right)+\mathrm{t}\left(\mathrm{CH}_{2}\right) \\
+\delta\left(\mathrm{NH}_{3}\right)+v(\mathrm{CC})\end{array}$ \\
\hline \multirow[t]{2}{*}{1070} & 1070 & 1112 & 1064 & 1036 & 1053 & 1062 & $\omega\left(\mathrm{CH}_{2}\right)+\mathrm{t}\left(\mathrm{CH}_{2}\right)$ \\
\hline & & & 1035 & & 1031 & & $\omega\left(\mathrm{CH}_{2}\right)+\mathrm{t}\left(\mathrm{CH}_{2}\right)$ \\
\hline 979 & 980 & 1018 & & & & & $v(\mathrm{CC})+v(\mathrm{CN})$ \\
\hline 915 & 915 & 970 & & & & & $\begin{array}{l}\mathrm{t}\left(\mathrm{CH}_{2}\right)+\delta_{\mathrm{s}}\left(\mathrm{NH}_{3}\right) \\
+\delta_{\text {sciss }}\left(\mathrm{NH}_{2}\right)+v(\mathrm{CC})\end{array}$ \\
\hline 884 & 865 & 906 & & & & & $\delta_{\text {sciss }}\left(\mathrm{NH}_{2}\right)+\omega\left(\mathrm{NH}_{2}\right)$ \\
\hline 843 & 841 & 880 & & & & & $\delta_{\mathrm{s}}\left(\mathrm{NH}_{3}\right)$ \\
\hline 766 & 772 & 766 & & & & & $\mathrm{r}\left(\mathrm{CH}_{2}\right)+\mathrm{r}\left(\mathrm{NH}_{3}\right)+\mathrm{r}\left(\mathrm{NH}_{2}\right)$ \\
\hline \multirow[t]{10}{*}{557} & 554 & & & & & & $\mathrm{r}\left(\mathrm{NH}_{3}\right)$ \\
\hline & & & 522 & $\begin{array}{l}536 \\
(524)^{b}\end{array}$ & & 522 & $\nu_{\mathrm{s}}(\mathrm{NMN})^{\mathrm{c}}$ \\
\hline & & & 483 & $\begin{array}{l}482 \\
(508)^{\mathrm{b}}\end{array}$ & 498 & 498 & $v_{\text {as }}(\mathrm{NMN})$ \\
\hline & & & & 391 & & 388 & $v(\mathrm{ClMCl})$ \\
\hline & & & & $\begin{array}{l}333 \\
(323)^{\mathrm{b}}\end{array}$ & & 340 & $\nu_{\mathrm{s}}(\mathrm{ClMCl})$ \\
\hline & & & & $\begin{array}{l}316 \\
(317)^{\mathrm{b}}\end{array}$ & & 307 & $v_{\mathrm{as}}(\mathrm{ClMCl})$ \\
\hline & 281 & & & & & & $\mathrm{LAM}^{\mathrm{d}}$ \\
\hline & & & & $\begin{array}{l}229 \\
(255)^{b}\end{array}$ & & 225 & $\delta(\mathrm{NMN})$ \\
\hline & & & & $\begin{array}{l}171 \\
(162)^{\mathrm{b}}\end{array}$ & & 173 & $\delta(\mathrm{ClMCl})$ \\
\hline & 118 & & & & & & $\mathrm{TAM}^{\mathrm{d}}$ \\
\hline
\end{tabular}

a At the B3LYP/6-31G** level wavenumbers above $600 \mathrm{~cm}^{-1}$ are scaled by 0.9614 [41]. $v$-Stretching; $\delta$-in-plane deformation; t-twisting; r-rocking; $\omega$-wagging; sciss-scissoring; s-symmetric; as-antisymmetric.

b From Ref. [31].

c $\mathrm{M}=\mathrm{Pt}(\mathrm{II})$ or $\mathrm{Pd}(\mathrm{II})$.

d LAM and TAM refer to longitudinal and transverse acoustic modes, respectively. 
Table 2

Main vibrational modes $\left(\mathrm{cm}^{-1}\right)$ for BENSpm and $\mathrm{Pd}_{2}-\mathrm{BENSpm}$.

\begin{tabular}{|c|c|c|c|c|c|}
\hline \multicolumn{3}{|c|}{ BENSpm } & \multirow{2}{*}{\multicolumn{2}{|c|}{$\frac{\mathrm{Pd}_{2} \text {-BENSpm }}{\text { Exp. }}$}} & \multirow[t]{3}{*}{ Approximate description } \\
\hline \multicolumn{2}{|l|}{ Exp. } & \multirow[t]{2}{*}{ Calc. ${ }^{a}$} & & & \\
\hline FTIR & Raman & & FTIR & Raman & \\
\hline 3147 & 3167 & 3342 & 3221 & 3229 & $v_{\mathrm{s}}\left(\mathrm{NH}_{2}\right)$ \\
\hline 3123 & & & & & $v_{\mathrm{s}}\left(\mathrm{NH}_{2}\right)$ \\
\hline 3083 & 3031 & 3028 & & & $v_{\mathrm{as}}\left(\mathrm{CH}_{3}\right)$ \\
\hline & & 3011 & & & $v_{\mathrm{as}}\left(\mathrm{CH}_{2}\right)+v_{\mathrm{as}}\left(\mathrm{CH}_{3}\right)$ \\
\hline 2989 & 2976 & 2975 & 2981 & 2992 & $v_{\text {as }}\left(\mathrm{CH}_{2}\right)$ \\
\hline 2951 & 2932 & 2947 & 2971 & 2968 & $v_{\mathrm{s}}\left(\mathrm{CH}_{2}\right)$ \\
\hline & & 2934 & & & $v_{\mathrm{s}}\left(\mathrm{CH}_{3}\right)$ \\
\hline 2927 & 2909 & 2923 & 2923 & 2923 & $v_{\mathrm{s}}\left(\mathrm{CH}_{2}\right)$ \\
\hline 2889 & 2881 & & 2865 & 2865 & $\nu v_{\mathrm{s}}\left(\mathrm{CH}_{2}\right)$ \\
\hline 1635 & 1694 & & 1625 & 1623 & $\delta_{\text {sciss }}\left(\mathrm{NH}_{2}\right)$ \\
\hline 1596 & 1595 & 1623 & & & $\delta_{\text {sciss }}\left(\mathrm{NH}_{2}\right)+\delta_{\text {sciss }}\left(\mathrm{CH}_{2}\right)$ \\
\hline 1490 & 1488 & 1511 & & & $\delta\left(\mathrm{CH}_{3}\right)$ \\
\hline 1461 & 1471 & 1500 & 1463 & 1455 & $\omega\left(\mathrm{CH}_{2}\right)+\mathrm{t}\left(\mathrm{CH}_{2}\right)+\omega\left(\mathrm{NH}_{2}\right)+\delta\left(\mathrm{NH}_{2}\right)$ \\
\hline 1405 & 1437 & 1468 & 1439 & 1433 & $\omega\left(\mathrm{CH}_{2}\right)+\mathrm{t}\left(\mathrm{CH}_{2}\right)+\omega\left(\mathrm{NH}_{2}\right)+\delta\left(\mathrm{NH}_{2}\right)$ \\
\hline 1394 & 1392 & 1456 & 1386 & 1388 & $\delta_{\text {sciss }}\left(\mathrm{CH}_{2}\right)+\mathrm{t}\left(\mathrm{NH}_{2}\right)$ \\
\hline 1357 & & 1405 & 1354 & 1355 & $\delta_{\text {sciss }}\left(\mathrm{CH}_{2}\right)+\mathrm{t}\left(\mathrm{CH}_{2}\right)+\omega\left(\mathrm{CH}_{2}\right)$ \\
\hline & 1312 & 1363 & 1306 & 1307 & $\omega\left(\mathrm{CH}_{2}\right)+\mathrm{t}\left(\mathrm{CH}_{2}\right)+\mathrm{t}\left(\mathrm{NH}_{2}\right)$ \\
\hline & & & 1296 & 1293 & $\omega\left(\mathrm{CH}_{2}\right)+\omega\left(\mathrm{NH}_{2}\right)+\delta\left(\mathrm{NH}_{2}\right)$ \\
\hline 1264 & 1257 & 1309 & 1281 & 1252 & $\omega\left(\mathrm{CH}_{2}\right)+\mathrm{t}\left(\mathrm{CH}_{2}\right)+\mathrm{t}\left(\mathrm{NH}_{2}\right)$ \\
\hline & & & 1175 & 1178 & $\omega\left(\mathrm{CH}_{2}\right)+\mathrm{r}\left(\mathrm{NH}_{2}\right)$ \\
\hline 1152 & 1149 & 1173 & 1146 & 1141 & $v(\mathrm{CC})+v(\mathrm{CN})$ \\
\hline 1050 & 1049 & 1084 & 1069 & 1074 & $v(\mathrm{CC})+v(\mathrm{CN})$ \\
\hline 1035 & 1035 & 1075 & 1038 & 1039 & $v(\mathrm{CC})+v(\mathrm{CN})$ \\
\hline & & & 1023 & 1014 & $v(\mathrm{CC})+v(\mathrm{CN})$ \\
\hline 999 & 999 & 1023 & 993 & 989 & $v(\mathrm{CC})+v(\mathrm{CN})$ \\
\hline & & & 966 & 936 & $\mathrm{t}\left(\mathrm{CH}_{2}\right)+\mathrm{r}\left(\mathrm{CH}_{2}\right)+\mathrm{r}\left(\mathrm{NH}_{2}\right)$ \\
\hline 906 & 884 & 931 & 892 & 887 & $\mathrm{t}\left(\mathrm{CH}_{2}\right)+\mathrm{r}\left(\mathrm{CH}_{2}\right)+\mathrm{r}\left(\mathrm{NH}_{2}\right)$ \\
\hline & & & 835 & 833 & $\mathrm{r}\left(\mathrm{NH}_{2}\right)$ \\
\hline 805 & 804 & 838 & 804 & 809 & $\mathrm{r}\left(\mathrm{CH}_{2}\right)+\mathrm{r}\left(\mathrm{NH}_{2}\right)$ \\
\hline 782 & 780 & 778 & 745 & 748 & $\mathrm{r}\left(\mathrm{CH}_{2}\right)+\mathrm{r}\left(\mathrm{NH}_{2}\right)$ \\
\hline & & & 697 & 700 & $\mathrm{r}\left(\mathrm{CH}_{2}\right)$ \\
\hline & & & & 563 & $v_{\mathrm{s}}(\mathrm{NPdN})$ \\
\hline & & & & 505 & $v_{\text {as }}(\mathrm{NPdN})$ \\
\hline & & & & 487 & $v_{\text {as }}(\mathrm{NPdN})$ \\
\hline & & & & 424 & $v_{\mathrm{s}}(\mathrm{ClPdCl})$ \\
\hline & & & & 325 & $v_{\mathrm{as}}(\mathrm{ClPdCl})$ \\
\hline & & & & 295 & $\delta(\mathrm{NPdN})$ \\
\hline & & & & 222 & $\delta(\mathrm{ClMCl})$ \\
\hline
\end{tabular}

${ }^{a}$ At the B3LYP/6-31G ${ }^{* *}$ level wavenumbers above $600 \mathrm{~cm}^{-1}$ are scaled by 0.9614 [41]. $v$-Stretching; $\delta$-in-plane deformation; t-twisting; r-rocking; $\omega$-wagging; sciss-scissoring; s-symmetric; as-antisymmetric.

compounds (BENSpm and CPENSpm) and between 1113 and $1126 \mathrm{~cm}^{-1}$ for NSpd; the $\mathrm{NH}_{2}$ rocking vibration, in turn, was observed at $745-970 \mathrm{~cm}^{-1}$ for the terminally alkylated polyamines, while for norspermidine a broad band, centred at $770 \mathrm{~cm}^{-1}$ was detected (particularly intense in infrared), encompassing both $\mathrm{NH}_{2}$ and $\mathrm{NH}_{3}$ rocking modes. For the alkylated molecules BENSpm and CPENSpm, a characteristic deformation mode of the terminal methyl group appears around $1480-1500 \mathrm{~cm}^{-1}$ (Tables 2 and 3). All the modified PA's presently studied are solids, except for NSpd which is a liquid at room temperature leading to an expected broadening of the FTIR and Raman signals (Fig. 2S, Supplementary Material).

The signals from the rocking deformations of the terminal $\mathrm{NH}_{3}$ groups, clearly detected for NSpd at $841 / 843 \mathrm{~cm}^{-1}$ by both FTIR (very intense band) and Raman (Table 1), are absent in BENSpm and CPENSpm due to alkylation at these nitrogen atoms (Figs. 2 and 3 ), while the bands assigned to $\mathrm{NH}_{2}$ symmetric and antisymmetric deformations are observed (as theoretically predicted) at ca. $1600 \mathrm{~cm}^{-1}$ for the three PA's. Although two calculated bands occur for these $\mathrm{NH}_{2}$ (scissoring) vibrations, ascribed to either the two inner amine groups or the terminal ones, the experimental data do not allow to distinguish them. Moreover, the NSpd $\mathrm{r}\left(\mathrm{NH}_{3}\right)$ modes are particularly intense in the FTIR spectra, as compared to the corresponding Raman profile (Fig. 2S, Supplementary Material), as expected. Also, the NH stretching vibrations display a larger infrared
Table 3

Main vibrational modes $\left(\mathrm{cm}^{-1}\right)$ for CPENSpm and $\mathrm{Pt}_{2}$-CPENSpm.

\begin{tabular}{|c|c|c|c|c|c|}
\hline \multicolumn{3}{|c|}{ CPENSpm } & \multirow{2}{*}{\multicolumn{2}{|c|}{$\frac{\mathrm{Pt}_{2}-\mathrm{CPENSpm}}{\text { Exp. }}$}} & \multirow[t]{3}{*}{ Approximate description } \\
\hline \multicolumn{2}{|l|}{ Exp. } & \multirow[t]{2}{*}{ Calc. $^{a}$} & & & \\
\hline FTIR & Raman & & FTIR & Raman & \\
\hline \multirow{2}{*}{$\begin{array}{l}3129 \\
3072\end{array}$} & & 3330 & 3139 & 3165 & $v_{\text {as }}\left(\mathrm{NH}_{2}\right)$ \\
\hline & 3070 & 3228 & & 3061 & $\nu_{\mathrm{s}}\left(\mathrm{NH}_{2}\right)$ \\
\hline 2973 & 2973 & $\begin{array}{l}3215 \\
3231\end{array}$ & 2960 & 2995 & $\begin{array}{l}v_{\text {as }}\left(\mathrm{CH}_{3}\right) \\
v_{3 \mathrm{~s}}\left(\mathrm{CH}_{2}\right)\end{array}$ \\
\hline \multirow[t]{3}{*}{2935} & 2934 & 3135 & 2937 & 2939 & $\nu \nu_{\mathrm{s}}\left(\mathrm{CH}_{2}\right)$ \\
\hline & 2907 & 3134 & & & $v(\mathrm{CH})^{\text {cyclop }}$ \\
\hline & & 3121 & & & $v_{\mathrm{s}}\left(\mathrm{CH}_{3}\right)$ \\
\hline \multirow[t]{2}{*}{2864} & 2878 & 2949 & 2879 & 2880 & $v_{\mathrm{s}}\left(\mathrm{CH}_{2}\right)$ \\
\hline & 2808 & 2885 & & & $v\left(\mathrm{CH} / \mathrm{CH}_{2}\right)^{\text {cyclop }}$ \\
\hline 1592 & & & 1608 & & $\delta_{\text {sciss }}\left(\mathrm{NH}_{2}\right)$ \\
\hline 1575 & 1577 & & & & $\delta_{\text {sciss }}\left(\mathrm{CH}_{2}\right)+\delta_{\text {sciss }}\left(\mathrm{NH}_{2}\right)$ \\
\hline 1481 & 1485 & 1500 & & & $\delta\left(\mathrm{CH}_{3}\right)$ \\
\hline 1452 & 1463 & & 1465 & 1461 & $\omega\left(\mathrm{CH}_{2}\right)+\mathrm{t}\left(\mathrm{CH}_{2}\right)+\delta\left(\mathrm{NH}_{2}\right)+\omega\left(\mathrm{NH}_{2}\right)$ \\
\hline 1408 & 1414 & 1477 & 1455 & 1451 & $\delta_{\text {sciss }}\left(\mathrm{CH}_{2}\right)$ \\
\hline 1391 & 1385 & 1464 & 1386 & 1373 & $\delta_{\text {sciss }}\left(\mathrm{CH}_{2}\right)$ \\
\hline \multirow[t]{2}{*}{1355} & 1349 & 1391 & & & $\delta_{\text {sciss }}\left(\mathrm{CH}_{2}\right)+\mathrm{t}\left(\mathrm{CH}_{2}\right)$ \\
\hline & 1314 & 1333 & 1320 & 1310 & $\omega\left(\mathrm{CH}_{2}\right)+\mathrm{t}\left(\mathrm{CH}_{2}\right)+\mathrm{t}\left(\mathrm{NH}_{2}\right)$ \\
\hline 1263 & 1256 & 1289 & & & $\omega\left(\mathrm{CH}_{2}\right)+\mathrm{t}\left(\mathrm{CH}_{2}\right)+\mathrm{t}\left(\mathrm{NH}_{2}\right)$ \\
\hline 1212 & 1201 & 1199 & 1191 & 1196 & $\omega\left(\mathrm{CH}_{2}\right)+\mathrm{t}\left(\mathrm{CH}_{2}\right)^{\text {cyclop }}+\mathrm{t}\left(\mathrm{NH}_{2}\right)$ \\
\hline 1148 & 1147 & 1175 & 1159 & & $\omega\left(\mathrm{CH}_{2}\right)+\mathrm{t}\left(\mathrm{NH}_{2}\right)$ \\
\hline 1049 & 1048 & 1104 & 1048 & & $v(\mathrm{CC})+v(\mathrm{CN})$ \\
\hline 1027 & & 1033 & & & $v v(\mathrm{CC})+v(\mathrm{CN})$ \\
\hline \multirow[t]{2}{*}{1000} & & 1013 & & & $v(\mathrm{CC})+v(\mathrm{CN})$ \\
\hline & 884 & 920 & 878 & & $\mathrm{t}\left(\mathrm{CH}_{2}\right)+\mathrm{r}\left(\mathrm{CH}_{2}\right)+\mathrm{t}\left(\mathrm{NH}_{2}\right)+\omega\left(\mathrm{NH}_{2}\right)$ \\
\hline 830 & 833 & 858 & 834 & 831 & $\mathrm{t}\left(\mathrm{CH}_{2}\right)+\mathrm{r}\left(\mathrm{CH}_{2}\right)+\mathrm{t}\left(\mathrm{NH}_{2}\right)$ \\
\hline 800 & 802 & 807 & 808 & 810 & $\mathrm{t}\left(\mathrm{CH}_{2}\right)^{\text {cyclop }}$ \\
\hline \multirow[t]{11}{*}{777} & 778 & 786 & 773 & 779 & $\mathrm{r}\left(\mathrm{CH}_{3}\right)+\mathrm{r}\left(\mathrm{NH}_{2}\right)$ \\
\hline & & & & 516 & $v_{\mathrm{s}}(\mathrm{NPtN})$ \\
\hline & & & & 483 & $\nu v_{\mathrm{as}}(\mathrm{NPtN})$ \\
\hline & & & & 386 & $v_{\mathrm{s}}(\mathrm{ClPtCl})$ \\
\hline & & & & 368 & $v_{s}(\mathrm{ClPtCl})$ \\
\hline & & & & 321 & $\nu v_{\text {as }}(\mathrm{ClPtCl})$ \\
\hline & 252 & & & & $v(\mathrm{CC})^{\text {cyclop }}$ \\
\hline & & & & 220 & $\delta(\mathrm{NPtN})$ \\
\hline & 216 & & & & LAM $^{\mathrm{b}}$ \\
\hline & & & & 197 & $\delta(\mathrm{ClPtCl})$ \\
\hline & & & & 168 & $\delta(\mathrm{ClPtCl})$ \\
\hline
\end{tabular}

a At the B3LYP/6-31G** level wavenumbers above $600 \mathrm{~cm}^{-1}$ are scaled by 0.9614 [41]. $v$-Stretching; $\delta$-in-plane deformation; t-twisting; r-rocking; $\omega$-wagging. sciss-scissoring; s-symmetric; as-antisymmetric.

b LAM refers to a longitudinal acoustic mode.

intensity relative to their Raman activity when compared to the $v(\mathrm{CH})$ modes, for all the molecules studied.

Unlike NSpd, which differs from Spd solely in the shorter carbon chain between nitrogens (three carbon atoms instead of four in the natural PA), the spectra of BENSpm and CPENSpm vary from those of their biogenic counterpart (spermine) in the absence of the terminal $\delta\left(\mathrm{NH}_{2}\right)$ mode at ca. $1600 \mathrm{~cm}^{-1}$, due to the terminal alkylation of these amines. It is interesting to note that the Raman spectrum of norspermidine displays quite strong features due to the stretching of the terminal $\mathrm{NH}_{3}$ groups, that are absent in the alkylated polyamines (for which the corresponding modes for the central $\mathrm{NH}_{2}$ moieties are undetectable). Furthermore, the cyclopropyl-containing PA, CPENSpm, displays several bands characteristic from this group (Table 3): the $\mathrm{CH}_{2}$ twisting modes, at $1201 \mathrm{~cm}^{-1}$, quite intense in the Raman spectrum (Table 3 ) while hardly observed by infrared; methylene deformations at $800 \mathrm{~cm}^{-1}$, present in both the FTIR and the Raman pattern (Fig. 3); and the ring C-C stretching, detected by Raman at $252 \mathrm{~cm}^{-1}$ (Fig. 1S, Supplementary Material). As opposed to norspermidine, the Raman bands corresponding to the amine stretching modes are not detected.

Some of the acoustic modes typical of this kind of all-trans, extended, molecules comprising repeating $\mathrm{CH}_{2} / \mathrm{NH}_{2}$ units $[23,26]$ - either longitudinal (LAM's) or transverse (TAM's) - were detected in the Raman spectra presently obtained (Tables 1 and 3), namely the symmetric LAM1 (accordion mode) at 281 and $216 \mathrm{~cm}^{-1}$ for NSpd 
and CPENSpm, respectively (Figs. 1S and 2S, Supplementary Material), and one of the lower frequency TAM's, observed for NSpd at $120 \mathrm{~cm}^{-1}$ (Fig. 2S, Supplementary Material). Additionally, NSpd and BENSpm being centrossymmetric species, a complementary infrared and Raman pattern was obtained, as opposed to their biogenic counterparts Spd and Spm [24,27].

The polyamine chelates presently studied vary in their coordination pattern, according to the type of ligand-either tri- or tetramine: in $\left(\mathrm{MCl}_{2}\right)_{3}(\mathrm{NSpd})_{2}\left(\mathrm{M}_{3}-\mathrm{NSpd}_{2}\right)$ two of the metal ions are bound to each of the norspermidine ligands, while the third is shared by both amines. In $\left(\mathrm{PdCl}_{2}\right)_{2}(\mathrm{BENSpm})\left(\mathrm{Pd}_{2}\right.$-BENSpm $)$ and $\left(\mathrm{PtCl}_{2}\right)_{2}(\mathrm{CPENSpm})$ ( $\mathrm{Pt}_{2}$-CPENSpm), in turn, the two metal centres coordinate to the four nitrogens of the spermine-like molecule.

The spectroscopic data presently gathered corroborate this type of coordination, both $\mathrm{Pt}(\mathrm{II})$ and $\mathrm{Pd}(\mathrm{II})$ being known to have a significant affinity for nitrogen atoms. Upon metal binding, the vibrational pattern of the ligands undergo clear changes, such as the expected loss of the main bands assigned to the $\mathrm{NH}_{2} / \mathrm{NH}_{3}$ groups, namely $\delta\left(\mathrm{NH}_{2}\right)$ around
$1600 \mathrm{~cm}^{-1}$, as well as $\rho\left(\mathrm{NH}_{3}\right)$ (Figs. 2 and 3; $3 \mathrm{~S}$ and $4 \mathrm{~S}$, Supplementary Material) (e.g. for NSpd, $\delta\left(\mathrm{NH}_{3}\right)$ at $c a .555 / 770 \mathrm{~cm}^{-1}$ and $\delta\left(\mathrm{NH}_{3}\right)$ at $c a$. $1120 \mathrm{~cm}^{-1}$ ). Additionally, the $\mathrm{NH}$ stretching bands reflect the presence of the metal through a marked shift to higher frequencies, plainly detected by FTIR for the terminally alkylated ligands BENSpm and CPENSpm (Figs. 2(b) and 3(b))-ascribed to the change from $v\left(\mathrm{NH}_{2}\right) /$ $v\left(\mathrm{NH}_{3}\right)$ symmetric and antisymmetric vibrations, in the free ligands, to the corresponding modes for the metal-coordinated amine groups. This is particularly evident in the Raman spectra of the terminally alkylated systems since the amine stretching features are hardly observable for the free ligands, but become quite intense for the corresponding complexes (Figs. 2(b) and 3(b)). For the non-alkylated polyamine $\mathrm{NSpd}$, in turn, these features $\left(v\left(\mathrm{NH}_{2}\right) / v\left(\mathrm{NH}_{3}\right)\right)$ are detected for both the complex and the uncoordinated molecule, undergoing a significant intensity decrease upon metal binding (Fig. 4S(b), Supplementary Material). Concomitantly, distinct new signals appear in the Raman pattern below $\mathrm{ca} .600 \mathrm{~cm}^{-1}$, characteristic of the metal coordination profile: deformations, between 165 and $225 \mathrm{~cm}^{-1}(\delta(\mathrm{Cl}-\mathrm{M}-\mathrm{Cl}))$ and 220 to (a)

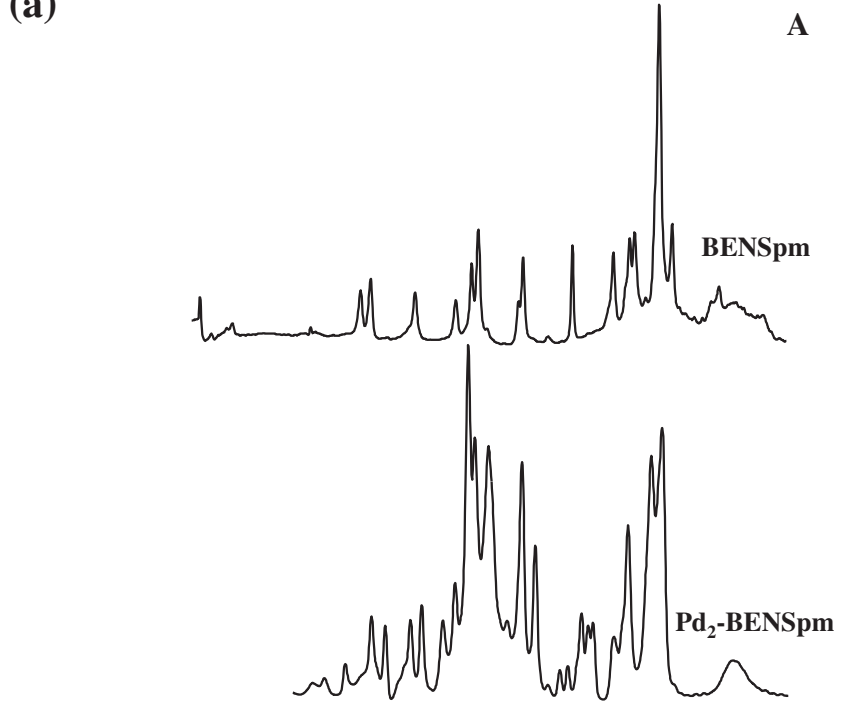

(b)

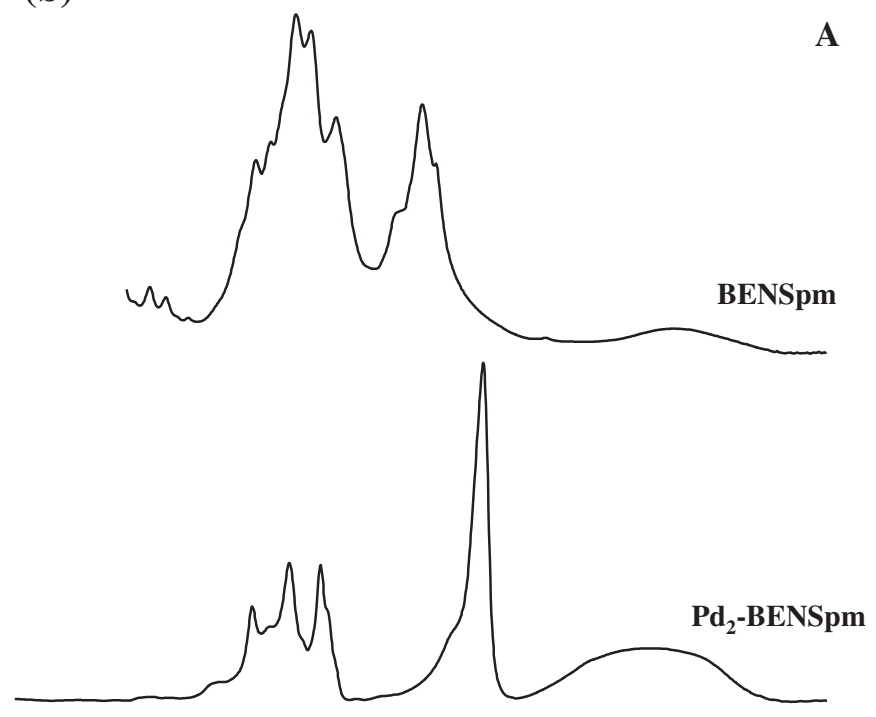

B

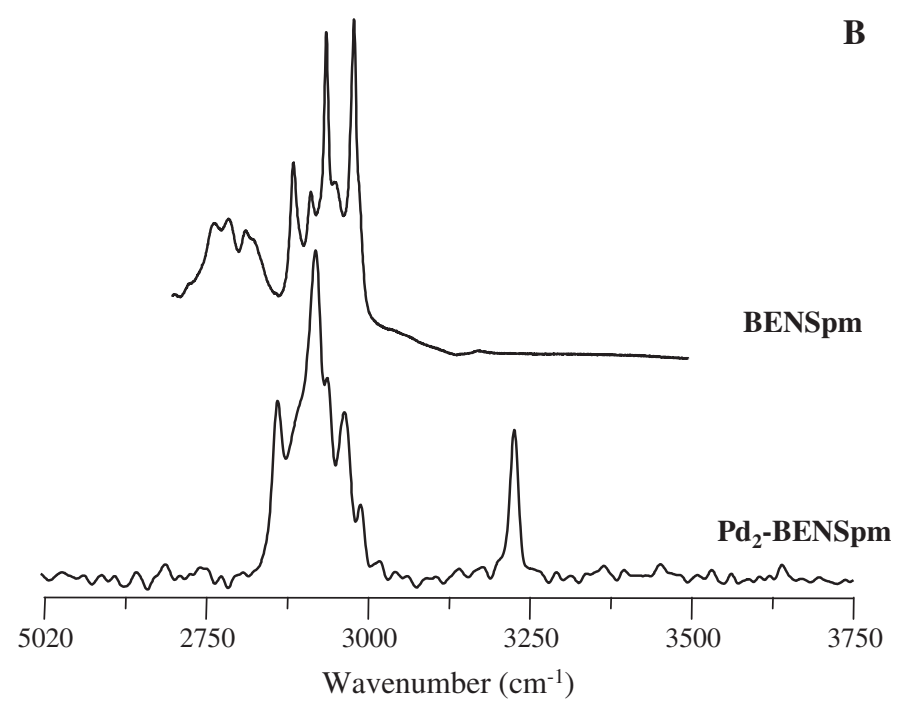

Fig. 2. Experimental FTIR (A) and Raman (B) spectra for BENSpm and $\mathrm{Pd}_{2}-\mathrm{BENSpm}$. 
(a)
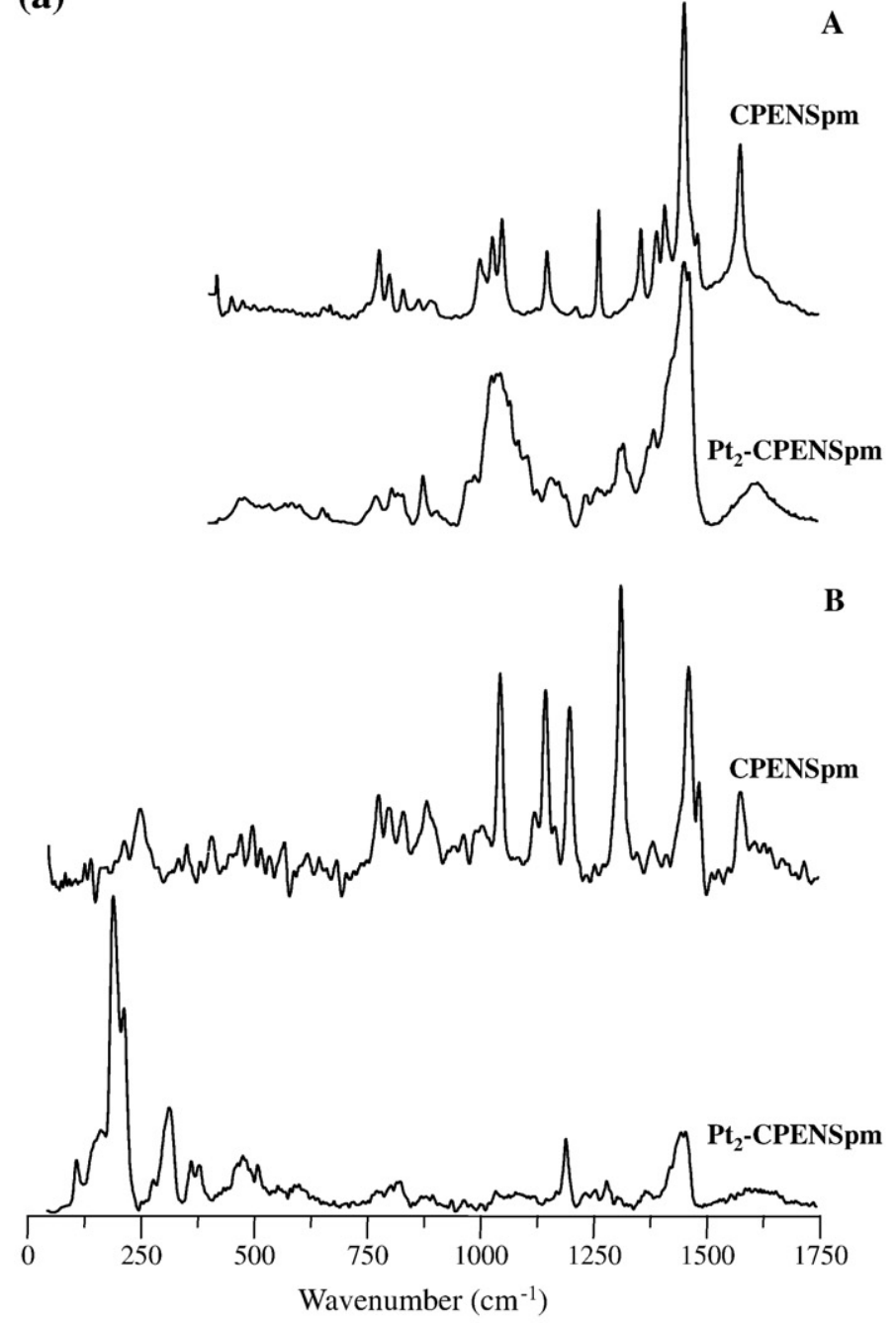

(b)
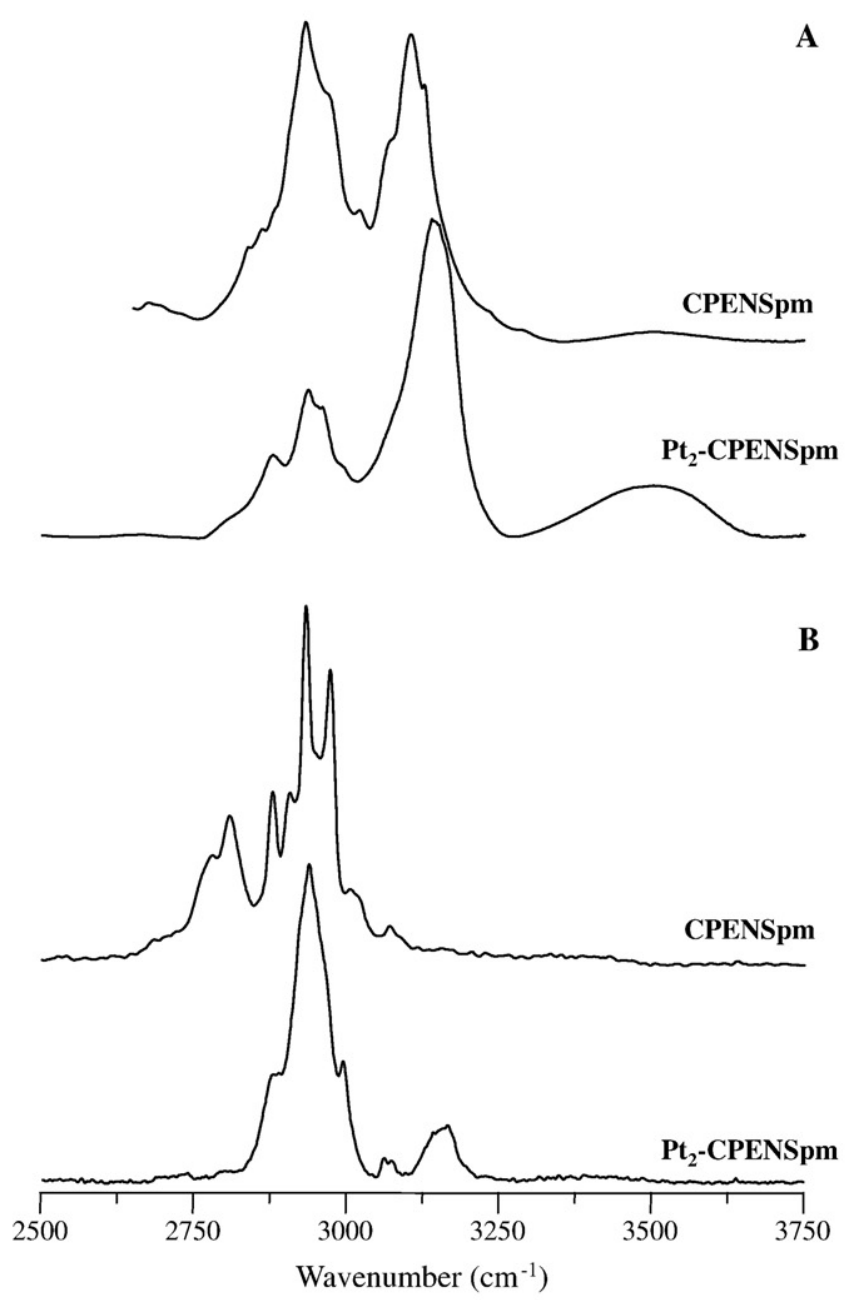

Fig. 3. Experimental FTIR (A) and Raman (B) spectra for CPENSpm and $\mathrm{Pt}_{2}-\mathrm{CPENSpm}$.

$295 \mathrm{~cm}^{-1}(\delta(\mathrm{N}-\mathrm{M}-\mathrm{N}))$, as well as symmetric and antisymmetric $(\mathrm{Cl}-\mathrm{M}-\mathrm{Cl})$ and $(\mathrm{N}-\mathrm{M}-\mathrm{N})$ stretching modes, between 305 to $425 \mathrm{~cm}^{-1}$ and 480 to $565 \mathrm{~cm}^{-1}$, respectively (Tables 1-3). A Pd for Pt substitution (in the NSpd complexes) was found to lead to a change in the band profile assigned to the symmetric and antisymmetric (metal-N) stretching modes-either a broad feature or a well resolved two-band pattern in the 460-540 $\mathrm{cm}^{-1}$ interval (Figs. 3S and 4S, Supplementary Material). Overall, complexation of the polyamine ligands leads to a marked weakening of the Raman bands at higher wavenumbers ( $c a$. above $1000 \mathrm{~cm}^{-1}$ ) relative to the very intense signals that appear below $\mathrm{ca}$. $600 \mathrm{~cm}^{-1}$, which are due to strong vibrational modes involving the metal and its primary coordination sphere.

These results are in accordance with the previously gathered data for cisplatin and its $\mathrm{Pd}$ (II) homologue [28-31], as well as for the similar polynuclear spermidine $\left(\mathrm{M}_{3}-\mathrm{Spd}_{2}\right)$ and spermine $\left(\mathrm{M}_{2}-\mathrm{Spm}\right)$ chelates $(\mathrm{M}=\mathrm{Pt}(\mathrm{II})$ or $\mathrm{Pd}(\mathrm{II}))$ [unpublished data]. Actually, comparison of the vibrational profile of the presently investigated cisplatin-like chelates, comprising two or three $\left(\mathrm{MCl}_{2} \mathrm{NH}_{2}\right)$ moieties, with cisplatin's vibrational pattern [31] allows to conclude that the deformation and stretching modes involving the metal centre, either platinum(II) or palladium(II), occur in defined spectral regions irrespective of the type of species - either the mononuclear cisplatin and its Pd(II) homologue, or the di- and trinuclear polyamine chelates - since they reflect a very similar metal coordination sphere (Table 1).

\section{Conclusions}

Interference with polyamine homeostasis through administration of modified homologues, and the use of metal complexes with a view to affect cell growth via DNA covalent binding are promising therapeutic approaches against cancer. In order to follow this type of strategies, a thorough knowledge of the conformational preferences of both modified polyamines and their chelates is crucial for understanding the mechanisms through which they are transported into the cell (pharmacokinetics) and the molecular basis of their biological activity (pharmacodynamics).

The reported results allow to conclude on the success of the synthetic route followed for the preparation of the $\mathrm{Pd}(\mathrm{II})$ and $\mathrm{Pt}(\mathrm{II})$ chelates with modified biogenic polyamines (tri- and tetramines), presently modified and improved relative to previously published methods applied to Spd and Spm complexes. Additionally, the theoretical approach used to simulate these systems showed to be quite suitable, as expected in the light of previous data published by the authors on cisplatin and analogous complexes (e.g. with Pd(II)).

As expected, and similarly to the previously reported studies on biogenic polyamines, the all-trans geometry was found to be energetically favoured for all the alkylated linear amines presently investigated, considering their physiological, totally protonated, state. As to the corresponding polynuclear chelates, newly synthesised through dedicated 
optimised routes, they display a stable geometry very similar to that previously obtained for their analogues with the biogenic PA's spermidine $\left(\mathrm{M}_{3}-\mathrm{Spd}_{2}\right)$ and spermine $\left(\mathrm{M}_{2}-\mathrm{Spm}\right)$, comprising two or three cisplatin-like $\left(\mathrm{MCl}_{2} \mathrm{NH}_{2}\right)$ moieties. Therefore, when interacting with DNA (their main biological target) they are able to bind to the purine/ pirimidine bases through two or three different sites simultaneously upon hydrolysis of the chloride atoms (long-range intra- and interstrand interactions), which can greatly enhance their cytotoxicity over conventional, mononuclear, drugs such as cisplatin.

This type of conformational studies (both for the targets and the potential chemotherapeutic agents) is the basis for a rational development of new anticancer drugs, coupling a higher (and hopefully selective) cytotoxic activity to an optimised therapeutic efficacy, as well as to the capacity of overcoming resistance to clinically used agents. Concomitant biological studies - cytotoxicity and cell growth inhibition evaluation - are being performed for both the modified PA's and their Pt(II) and $\mathrm{Pd}(\mathrm{II})$ chelates in human cancer cell lines (e.g. breast cancer), aiming at linking structural preferences to anticancer effect, leading to the establishment of consistent structure-activity relationships (SAR's) for this kind of polyamine-based systems.

\section{Abbreviations}

$\begin{array}{ll}\text { Put } & \text { putrescine } \\ \text { Spd } & \text { spermidine } \\ \text { Spm } & \text { spermine } \\ \text { NSpd } & \text { norspermidine } \\ \text { BENSpm } & N^{1}, N^{11} \text {-bis(ethyl)norspermine } \\ \text { CPENSpm } & N^{1} \text {-cyclo-propylmethyl- } N^{11} \text {-ethylnorspermine } \\ \text { PA } & \text { polyamines } \\ \text { FTIR } & \text { Fourier transform infrared spectroscopy } \\ \text { MCT } & \text { mercury cadmium telluride } \\ \text { CCD } & \text { charge coupled device } \\ \text { DFT } & \text { density functional theory } \\ \text { HF } & \text { Hartree-Foch } \\ \text { rms } & \text { root-mean-square }\end{array}$

Supplementary data to this article can be found online at doi:10. 1016/j.jinorgbio.2011.11.021.

\section{Acknowledgements}

The authors thank the Chemistry Department of the University of Aveiro for use of the FT-Raman spectrometer. The authors acknowledge financial support from the Portuguese Foundation for Science and Technology-PEst-OE/QUI/UI0070/2011 and PhD fellowship SFRH/BD/46364/2008 (TMS). TMS is grateful for help regarding the synthetic procedure (Sónia Fiuza) and spectral analysis (Nelson Machado).

\section{References}

[1] K. Igarashi, K. Kashiwagi, Plant Physiol. Biochem. 48 (2010) 506-512.

[2] E. Agostinelli, M.P. Marques, R. Calheiros, F.P. Gil, G. Tempera, N. Viceconte, V. Battaglia, S. Grancara, A. Toninello, Amino Acids 38 (2010) 393-403.

[3] A. Onal, Crit. Rev. Anal. Chem. 40 (2010) 60-67.

[4] K. Dredge, J.A. Kink, R.M. Johnson, I. Bytheway, L.J. Marton, Cancer Chemother. Pharmacol. 65 (2009) 191-195.

[5] N. Seiler, Curr. Drug Targets 4 (2003) 565-585.

[6] R.A. Casero, P.M. Woster, J. Med. Chem. 52 (2009) 4551-4573.
[7] R.A. Casero, P.M. Woster, J. Med. Chem. 44 (2001) 1-26.

[8] Y. Huang, A. Pledgie, R.A. Casero Jr., N.E. Davidson, Anticancer Drugs 16 (2005) 229-241.

[9] A.C. Wolff, D.K. Armstrong, J.H. Fetting, M.K. Carducci, C.D. Riley, J.F. Bender, R.A. Casero Jr., N.E. Davidson, Clin. Cancer Res. 9 (2003) 5922-5928.

[10] Pledgie-Tracy, M. Billam, A. Hacker, M.D. Sobolewski, P.M. Woster, Z. Zhang, R.A. Casero, N.E. Davidson, Cancer Chemother. Pharmacol. 65 (2010) 1067-1081.

[11] K. Chválová, J. Kaspárková, N. Farrell, V. Brabec, FEBS J. 273 (2006) 3467-3478.

[12] S. Komeda, T. Moulaei, M. Chikuma, A. Odani, R. Kipping, N. Farrell, L.D. Williams, Nucleic Acids Res. (2010) 1-12.

[13] M.P. Marques, T. Girão, M.C. Pedroso de Lima, A. Gameiro, E. Pereira, P. Garcia, Biochim. Biophys. Acta-MCR 1589 (2002) 63-70.

[14] L.J. Teixeira, M. Seabra, E. Reis, M.T. da Cruz, M.C. de Lima, E. Pereira, M.A. Miranda, M.P. Marques, J. Med. Chem. 47 (2004) 2917-2925.

[15] S.M. Fiuza, A.M. Amado, Paulo J. Oliveira, Vilma A. Sardão, L.A.E. Batista de Carvalho, M.P. Marques, Lett. Drug Des. Discov. 3 (2006) 149-151.

[16] A.S. Soares, S.M. Fiuza, M.J. Gonçalves, L.A.E. Batista de Carvalho, M.P. Marques, A.M. Urbano, Lett. Drug Des. Discov. 4 (2007) 460-463.

[17] R. Tummala, P. Diegelman, S.M. Fiuza, L.A.E. Batista de Carvalho, M.P. Marques, D.L. Kramer, K. Clark, S. Vujcic, C.W. Porter, L. Pendyala, Oncol. Rep. 24 (2010) $15-24$.

[18] A. Tassoni, N. Bagni, M. Ferri, M. Franceschetti, A. Khomutov, M.P. Marques, S.M. Fiuza, A.R. Simonian, D. Serafini-Fracassini, Plant Physiol. Biochem. 48 (2010) 496-505.

[19] O. Corduneanu, A.M. Chiorcea-Paquim, S.M. Fiuza, M.P. Marques, A.M. OliveiraBrett, Bioelectrochemistry 78 (2010) 97-105.

[20] O. Corduneanu, A.M. Chiorcea-Paquim, V. Diculescu, S.M. Fiuza, M.P. Marques, A.M. Oliveira-Brett, Anal. Chem. 82 (2010) 1245-1252.

[21] S.M. Fiuza, J. Holy, L.A.E. Batista de Carvalho, M.P. Marques, Chem. Biol. Drug Des. 77 (2011) 477-488.

[22] A.M. Amorim da Costa, M.P. Marques, L.A.E. Batista de Carvalho, Vib. Spec. 29 (2002) 61-67.

[23] M.P. Marques, L.A.E. Batista de Carvalho, J. Tomkinson, J. Phys. Chem. A 106 (2002) 2473-2482

[24] A.M. Amorim da Costa, M.P. Marques, L.A.E. Batista de Carvalho, J. Raman Spectrosc. 34 (2003) 357-366

[25] A.M. Amorim da Costa, L.A.E. Batista de Carvalho, M.P.M. Marques, Vib. Spec. 35 (2004) 165-171.

[26] L.A.E. Batista de Carvalho, M.P. Marques, J. Tomkinson, J. Phys. Chem. A 110 (2006) 12947-12954

[27] M.P. Marques, L.A.E. Batista de Carvalho, Biochem. Soc. Trans. 35 (2007) 374-380.

[28] A.M. Amado, S.M. Fiuza, M.P. Marques, L.A.E. Batista de Carvalho, J. Chem. Phys. 127 (2007) 185104-185110.

[29] S.M. Fiuza, A.M. Amado, M.P. Marques, L.A.E. Batista de Carvalho, J. Phys. Chem. A 112 (2008) 3253-3259.

[30] S.M. Fiuza, A.M. Amado, H.F. dos Santos, M.P. Marques, L.A.E. Batista de Carvalho, Phys. Chem. Chem. Phys. 12 (2010) 14309-14321.

[31] L.A.E. Batista de Carvalho, M.P. Marques, C. Martin, S.F. Parker, J. Tomkinson, Chem. Phys. Chem. 12 (2011) 1334-1341.

[32] C. Navarro-Ranninger, P.A. Ochoa, J.M. Pérez, V.M. González, J.R. Masaguer, C. Alonso, J. Inorg. Biochem. 53 (1994) 177-190.

[33] G. Codina, A. Caubet, C. Lopez, V. Moreno, E. Molins, Helv. Chim. Acta 82 (1999) 1025-1037.

[34] M.J. Frisch, G.W. Trucks, H.B. Schlegel, G.E. Scuseria, M.A. Robb, J.R. Cheeseman, J.A. Montgomery Jr., T. Vreven, K.N. Kudin, J.C. Burant, J.M. Millam, S.S. Iyengar, J. Tomasi, V. Barone, B. Mennucci, M. Cossi, G. Scalmani, N. Rega, G.A. Petersson, H. Nakatsuji, M. Hada, M. Ehara, K. Toyota, R. Fukuda, J. Hasegawa, M. Ishida, T. Nakajima, Y. Honda, O. Kitao, H. Nakai, M. Klene, X. Li, J.E. Knox, H.P. Hratchian, J.B. Cross, V. Bakken, C. Adamo, J. Jaramillo, R. Gomperts, R.E. Stratmann, O. Yazyev, A.J. Austin, R. Cammi, C. Pomelli, J. Ochterski, P.Y. Ayala, K. Morokuma, G.A. Voth, P. Salvador, J.J. Dannenberg, V.G. Zakrzewski, S. Dapprich, A.D. Daniels, M.C. Strain, O. Farkas, D.K. Malick, A.D. Rabuck, K. Raghavachari, J.B. Foresman, J.V. Ortiz, Q. Cui, A.G. Baboul, S. Clifford, J. Cioslowski, B.B. Stefanov, G. Liu, A. Liashenko, P. Piskorz, I. Komaromi, R.L. Martin, D.J. Fox, T. Keith, M.A. Al-Laham, C.Y. Peng, A. Nanayakkara, M. Challacombe, P.M.W. Gill, B.G. Johnson, W. Chen, M.W. Wong, C. Gonzalez, J.A. Pople, G. GAUSSIAN 03, Revision D.01 Gaussian, Inc., Wallingford, CT, 2004.

[35] C. Lee, W. Yang, R.G. Parr, Phys. Rev. B 37 (1988) 785.

[36] B. Miehlich, A. Savin, H. Stoll, H. Preuss, Chem. Phys. Lett. 157 (1989) 200-206.

[37] A.D. Becke, Phys. Rev. A 38 (1988) 3098-3100.

[38] A.D. Becke, J. Chem. Phys. 98 (1993) 5648-5652.

[39] G.A. Petersson, A. Bennett, T.G. Tensfeldt, M.A. Al-Laham, W.A. Shirley, J. Mantzaris, J. Chem. Phys. 89 (1988) 2193.

[40] C. Peng, P.Y. Ayala, H.B. Schlegel, M.J. Frisch, J. Comput. Chem. 17 (1996) 49.

[41] J.P. Merrick, D. Moran, L. Radom, J. Phys. Chem. A 111 (2007) 11683-11700.

[42] L.A.E. Batista de Carvalho, L.E. Lourenço, M.P. Marques, J. Mol. Struct. 639 (1999) 482-483. 